\title{
Reconstruction of Atomic Resolution STEM Images Using the Diffraction-Imaging Method with an Aberration-Corrected STEM.
}

\author{
Eiji Okunishi, Hidetaka Sawada and Yukihito Kondo
}

EM business unit JEOL Ltd., 1-2 Musashino 3-chome Akishima Tokyo, 196-8558, Japan.

High angle annular dark field (HAADF) imaging by aberration-corrected scanning transmission electron microscopes (STEMs) has been well established as a method for direct observation of atomic arrangement in atomic resolution. Recently, annular bright field (ABF) imaging was found as a new technique of visualization of atomic sites of both light and heavy elements simultaneously [1, 2]. For the determination of types of element, energy dispersive X-ray spectrometry (EDS) or electron energy loss spectrometry (EELS) is usually used with STEM and the spatial resolution has reached an atomic revel [3]. The Diffraction-Imaging method has recently been developed and commercialized [4]. This method enables us to record each electron diffraction patterns projected on a CCD camera from each individual pixel in a scanned area of STEM. It has so far been used as a domain observation technique [5], which detects magnetization of ferro-magnetic materials or di-polization of ferro-electric materials. In this presentation, we report the observation of diffraction patterns from single atomic site columns and the reconstruction of three types of atomic resolution STEM images using all the diffraction patterns from all the scanned pixels.

The experiment was performed with JEM-ARM200F: an aberration-corrected STEM, equipped with the GIF-QuantumER imaging filter (GATAN). This experimental arrangement enables us to acquire annular dark field (ADF) signals and diffraction patterns simultaneously. The specimen for the present experiment was $\mathrm{SrTiO}_{3}(001)$ prepared by Ar ion milling. All diffraction patterns were taken the 2k x 2k CCD camera (Gatan) attached to the GIF-QuantumER imaging filter. The probe size and current for the present experiment were set to be $0.1 \mathrm{~nm}$ and $20 \mathrm{pA}$ with a convergence semi angle of $22 \mathrm{mrad}$. The camera length was adjusted to cover an angular range from 0 to $80 \mathrm{mrad}$ on CCD. Exposure time to record each diffraction pattern from each individual pixel was $100 \mathrm{~ms}$.

Fig. 1 shows nine diffraction patterns from three kind of atomic site columns: $\mathrm{Sr}, \mathrm{Ti}+\mathrm{O}, \mathrm{O}$. Each diffraction pattern shows certain symmetric contrast. The contrast from same type of column is similar to each other whereas the contrast from the different type of column is different. For instance, the contrast at the center from the $\mathrm{O}$ columns bright whereas that from other two types of column is dark. Figs. 2(d), 2(e) and 2(f) show high resolution STEM images reconstructed from all scanned pixels from all the diffraction patterns in three areas with different scattering angles on the back focal plane shows in Fig. 2(b). Each atomic position in reconstructed images well coincides with that in the HAADF image shown in Fig. 2(c). The Sr and Ti+O site columns show dark contrast in the bright-field image (Fig. 2(d)) reconstructed from the area 1 shown in fig. 2(b) whereas they show bright contrast in the ADF image (Fig. 2(e)) reconstructed from area 3. There is extra slightly dark contrast at the $\mathrm{Sr}$ and $\mathrm{Ti}+\mathrm{O}$ site columns in the $\mathrm{ABF}$ image (Fig. 2(e)) reconstructed from area 2. The position with this contrast corresponds to the $\mathrm{O}$ site column. Reconstructed atomic resolution STEM images were obtained using the Diffraction imaging method.

References

[1] E. Okunishi et al, proc. M\&M 2009

[2] S. findley et al, Appl. Phys. Lett. 10, 6, (2009) 997.

[3]M. Watanabe et al, JEOL news, vol45, 2010 
[4] www.gatan.com. , GATAN web site

[5] A. Yasuhara et al, M\&M 2009

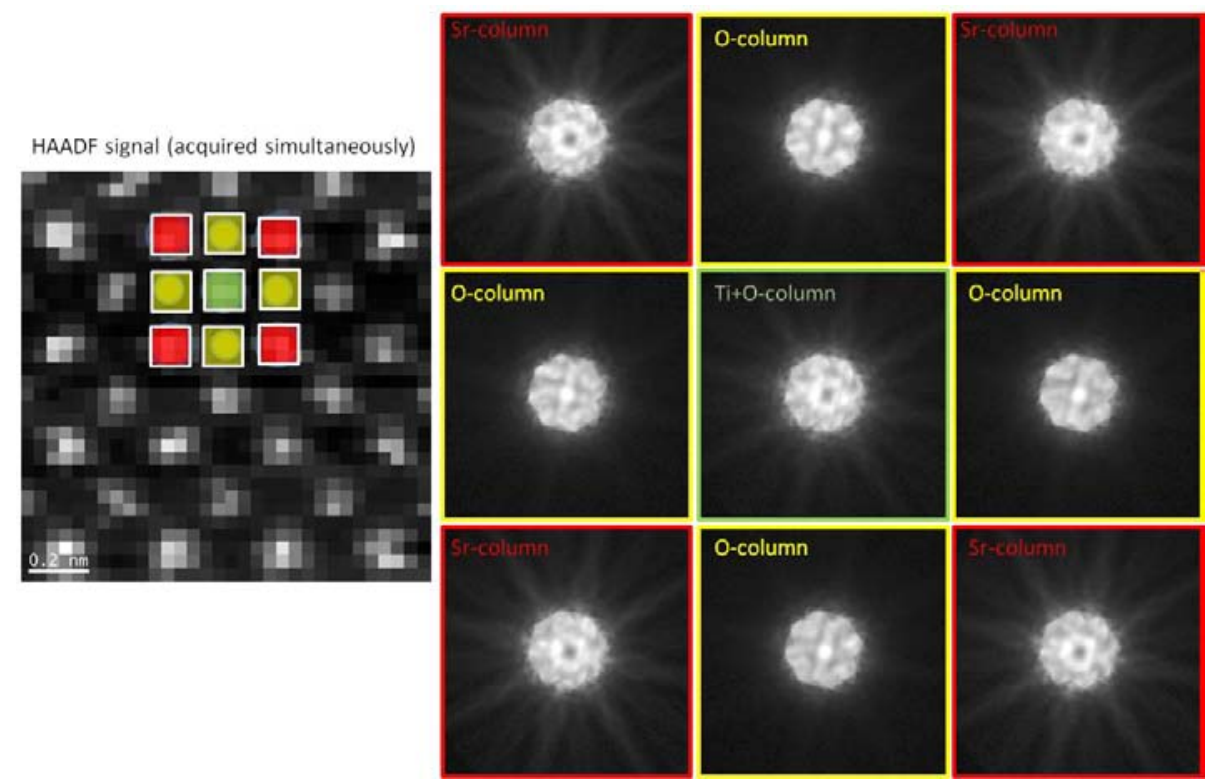

Fig. 1. Diffraction patterns from nine atomic site columns. Each diffraction pattern were extracted from "Diffraction Image Data Cube"
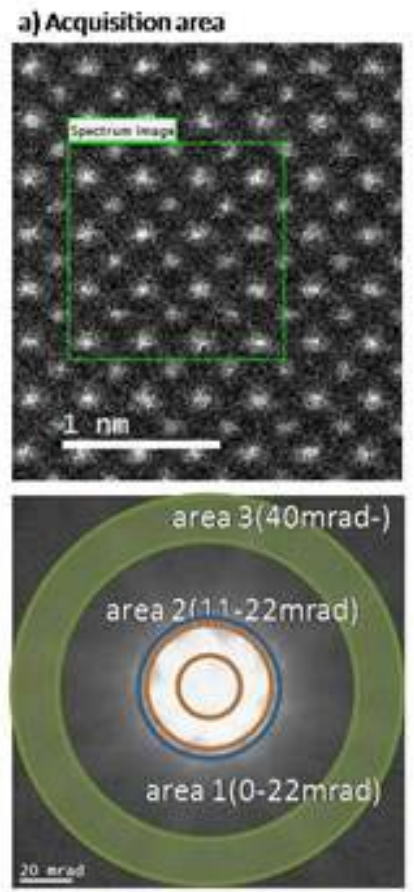

b) Diffraction pattern at all region

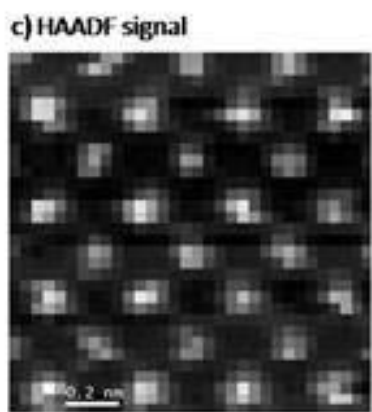

e) Reconstruct image by area 2

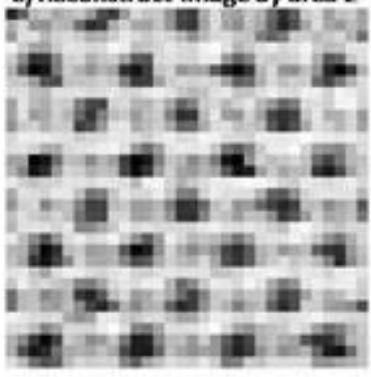

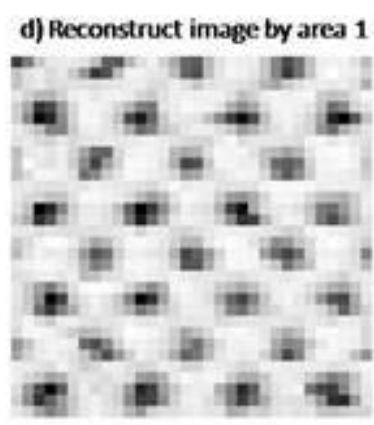

f) Reconstruct image by area 3

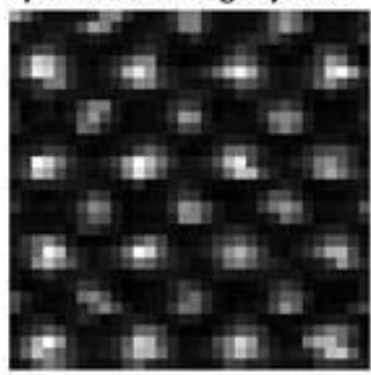

FIG. 2. Reconstructed HR-STEM images from three annular regions with different scattering angles in diffraction space.

a) A marked rectangular area was selected for acquiring diffraction patterns.

b) The area on the back focal plane where the diffraction pattern were recorded.

c) The HAADF image of the selected area. It was acquired simultaneously with the diffraction patterns.

d)-f) Reconstructed HR-STEM images) BF image, e)ABF image and f) ADF image 